\title{
A Review of Mixed Strain Clostridium difficile Colonization and Infection
}

\author{
Pete Dayananda ${ }^{1,2}$ and Mark H. Wilcox ${ }^{1,2 *}$ \\ ${ }^{1}$ Department of Microbiology, Leeds Teaching Hospitals NHS Trust, Leeds, United Kingdom, ${ }^{2}$ Leeds Institute of Biomedical \\ and Clinical Sciences, University of Leeds, Leeds, United Kingdom
}

\section{OPEN ACCESS}

Edited by:

Uwe Groß,

University Medical Center Göttingen,

Germany

Reviewed by:

John F. Kokai-Kun,

Synthetic Biologics, Inc.,

United States

Stuart Johnson,

Loyola University Chicago,

United States

*Correspondence:

Mark H. Wilcox

mark.wilcox@nhs.net

Specialty section:

This article was submitted to

Antimicrobials, Resistance

and Chemotherapy,

a section of the journal

Frontiers in Microbiology

Received: 23 July 2018

Accepted: 19 March 2019

Published: 10 April 2019

Citation:

Dayananda $P$ and Wilcox $\mathrm{MH}$ (2019) A Review of Mixed Strain Clostridium difficile Colonization and Infection.

Front. Microbiol. 10:692. doi: 10.3389/fmicb.2019.00692
Given that Clostridium difficile is not part of the normal human microbiota, if multiple strains are to accumulate in the colon implies successive exposure events and/or persistent colonization must occur. Evidence of $C$. difficile infection (CDI) with more than one strain was first described in 1983. Despite the availability of increasingly discriminatory bacterial fingerprinting methods, the described rate of dual strain recovery in patients with $\mathrm{CDI}$ has remained stable at $\sim 5-10 \%$. More data are needed to determine when dual strain infection may be harmful. Notably, one strain may block the establishment of and infection by another. In humans, patients colonized by nontoxigenic C. difficile strain are at a lower risk of developing CDI. Further studies to elucidate the interaction between co-infecting or colonizing and infecting $C$. difficile strains may help identify potential exploitable mechanisms to prevent CDI.

Keywords: carriage, competition, microbiome, antibiotic, colon

\section{INTRODUCTION}

Clostridium (Clostridioides) difficile is an anaerobic, spore forming, Gram-positive bacillus (Martin et al., 2016). Historically, C. difficile infection (CDI) primarily occurs in hospitalized patients secondary to antibiotic use (Kelly and LaMont, 1998). However, in the past decade, the proportion of C. difficile infection occurring in the community (previously thought to be low risk) is increasing (DePestel and Aronoff, 2013). This highlights the endemicity of C. difficile outside healthcare institutions. In addition, the emergence of a more virulent $C$. difficile strain BI/NAP1/027 changed perceptions of $C$. difficile from an easily treated side-effect of antibiotic use to a leading cause of infectious diarrhea with increased morbidity and mortality worldwide (Baines et al., 2013; Ghose, 2013; Sun et al., 2016).

There are over 800 recognized strain types (ribotypes) of $C$. difficile and only toxin-producing strains are associated with disease (Tonna and Welsby, 2005; Vedantam et al., 2012). C. difficile associated diarrhea is mediated by the production of toxin A (TcdA) and toxin B (TcdB) released into the gut as a result of colonization by toxigenic strains (van den Berg et al., 2005). Early hamster models suggested that TcdA is a key determinant of intestinal inflammation (Lyerly et al., 1985), but subsequent studies suggested that TcdB is more potent (Savidge et al., 2003; Lyras et al., 2009). Furthermore, a monoclonal anti-toxin B antibody, but not an anti-toxin A antibody, was effective at reducing recurrence in patients treated for CDI (Wilcox et al., 2017). Also, there is a growing body of evidence for TcdA negative/TcdB positive CDI cases (Samra et al., 2002; Carter et al., 2015; 
Di Bella et al., 2016). Therefore, the importance of TcdA in human beings remains uncertain and needs further clarification. Since the emergence of the more virulent BI/NAP1/027 strain of $C$. difficile, the role of a third binary toxin, $C$. difficile transferase (CDT) is increasingly recognized for its association with enhanced virulence and higher patient mortality (Gerding et al., 2014; Berry et al., 2017).

The method used for diagnosing CDI is one of the factors limiting detection of multiple $C$. difficile strains in health or disease. CDI diagnosis is based ideally on detection of free fecal toxin, or, with less specificity, by the presence of toxin genes (Surawicz et al., 2013; Martin et al., 2016). Therefore, in the majority of settings, $C$. difficile culture is not performed and so the number of $C$. difficile strains present is not determined.

The likelihood of detecting multi-strain infection or colonization varies with the methods used. Multiple strains can be detected by methods that can distinguish individual strains. In the context of $C$. difficile, these methods include restriction enzyme analysis (REA), pulsed field gel electrophoresis (PGFE), PCR ribotyping, multilocus variable number tandem repeat analysis (MLVA), multilocus sequence typing (MLST), and whole genome sequencing (WGS) (Tenover et al., 2011; Knetsch et al., 2013; Sim et al., 2017). Multiple strains may be detected by the presence of more alleles present at a particular locus than is possible if just one strain is present, dissimilar genotypes from different colonies grown from the same isolate or difference in ability to produce cytotoxin (Borriello and Honour, 1983; Balmer and Tanner, 2011). Notably, the limited studies that have investigated the presence of multiple strains of $C$. difficile in patients with CDI, differ in their case selection criteria and in the methods used for $C$. difficile culture, the number of colonies tested and differentiation of strains (often reflecting the available diagnostic technology) (Table 1).

\section{SIGNIFICANCE}

A key issue regarding colonization or infection by more than one strain of $C$. difficile is the determination of whether recurrence is due to the same (relapse) or different (reinfection) strain. Most such CDI recurrence studies have examined single or few colonies, or have not used a highly discriminative method, both of which reduce the chance of isolating multiple strains of $C$. difficile. For example, Figueroa et al. (2012) examined 90 patients who had recurrent $C$. difficile infection using REA on single $C$. difficile colonies (Figueroa et al., 2012). They showed that 75 participants $(83.3 \%)$ had a relapse. The remaining 15 (16.7\%) participants were found to have a reinfection. Based on the results obtained, there was no evidence to suggest the presence of concomitant carriage of more than 1 strain of C. difficile (Figueroa et al., 2012) Although REA has respectable discriminatory power, newer methods such as capillary PCR ribotyping and MLVA are more discriminatory and therefore have a higher chance of identifying mixed C. difficile strain infections (Kuijper et al., 2009). Kamboj et al. (2011) used PCR ribotyping to explore patients with recurrent $C$. difficile infection. The results suggested that the majority of patients with recurrent $C$. difficile infection within
8 weeks (85/102 patients) had a relapse and not a reinfection. This method has similar discriminative power to REA (Kuijper et al., 2009). Based on the results, there was no evidence to suggest the presence of more than 1 strain of $C$. difficile at any one time (Kamboj et al., 2011). This may be due to the small number of colonies studied as per the published protocol (Bidet et al., 1999). A recent study by Behroozian et al. (2013) recognized this limitation and analyzed approximately 95 colonies per sample and found evidence of more than 1 C. difficile ribotype in 16/102 (16\%) cases. Even with the large number of colonies studied, there is a chance that less abundant ribotypes will be overlooked (Behroozian et al., 2013).

As C. difficile is ubiquitous in nature, the presence of multiple strains could simply reflect a recently ingested strain when another has already colonized or been newly ingested. However, colonization by multiple strains could affect the level of host protection against $C$. difficile infection. At present, there is insufficient evidence to determine the full implications of colonization/infection with more than 1 strain of $C$. difficile. Baines et al. (2013) demonstrated using an in vitro gut model that 2 different population of $C$. difficile (differentiated by antimicrobial susceptibility) were able to concurrently colonize, populate and produce toxin. It is however, not possible to determine the degree of contribution toward toxin production (Baines et al., 2013). Longitudinal studies are needed to determine the significance of this phenomenon in human beings.

Data from murine models suggest that colonization with a non-toxigenic strain of $C$. difficile protects against disease in hamster following a challenge with a toxigenic strain (Merrigan et al., 2009). Balmer et al. suggests that in human beings, infection with more than 1 strain of $C$. difficile is likely to be a significant clinical and immunological phenomenon as it may overwhelm the immune system by influencing the host immune response in different ways. This may also affect pathogen evolution, potentiate competitive, or mutualistic pathogenpathogen interaction, horizontal gene flow and treatment options compared with single strain infection (Balmer and Tanner, 2011). On the other hand, the presence of two competing strains can be beneficial to the host as they could control each other, similar to how probiotics have been proposed for the prevention of CDI (Goldenberg et al., 2013). Notably, Gerding et al. demonstrated that patients with an episode of primary CDI or first recurrence within 8 weeks of the primary episode benefitted from colonization with non-toxigenic $C$. difficile strain M3 (NTCD-M3). The recurrence rate of CDI in patients colonized with NTCD-M3 was significantly lower compared with patients who were not (11 vs. $30 \%)$. This reaffirms the notion that colonization with a non-toxigenic strain is beneficial to the host (Gerding et al., 2015).

\section{DUAL STRAIN INFECTION}

The observed incidence of mixed $C$ difficile toxigenic strain infection has been relatively stable over recent decades at approximately 7-16\% of all cases (Eyre et al., 2012, 2013; Behroozian et al., 2013; Sun et al., 2016). Evidence of mixed strain 


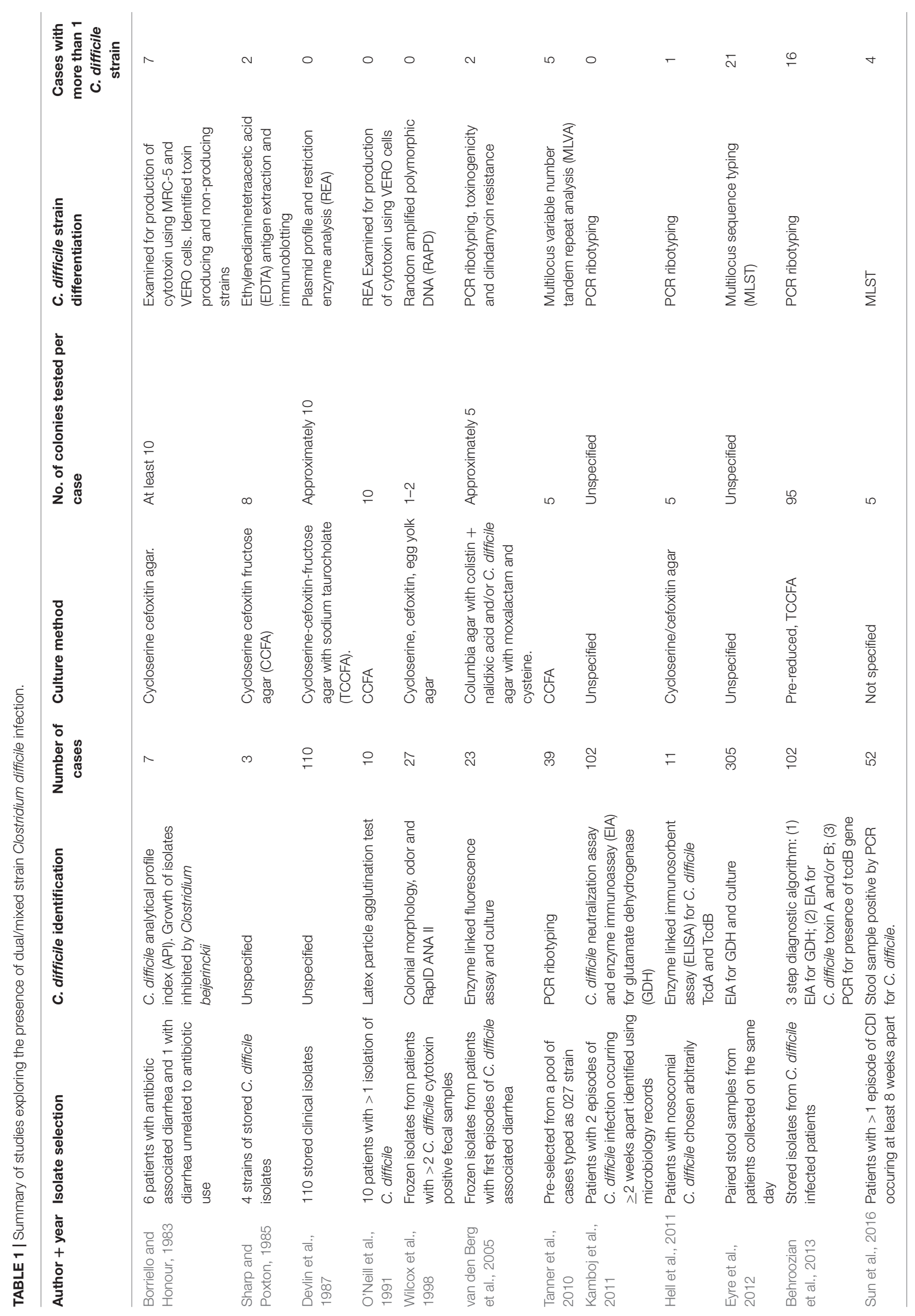


infection was first reported by Borriello and Honour (1983). The authors found that stool samples from all 6 studied cases showed isolates of $C$. difficile that differed in cytotoxin production (Borriello and Honour, 1983). O'Neill et al. (1991) later reported a patient who had suffered both a reinfection and relapse. The isolated strain did not produce cytotoxin in vitro but toxin was detected in the stools. They hypothesized that this is due to the presence of concomitant strains of C. difficile. However, further investigation on 10 different colonies from each sample using REA and cytotoxin studies did not support this (O’Neill et al., 1991). Similarly, in a larger study, Wilcox et al. (1998) retrospectively analyzed C. difficile colonies using random amplification of polymorphic DNA (RAPD) fingerprinting, but did not identify show more than one $C$. difficile strain per sample time point (Wilcox et al., 1998). This may be due to the fact that mixed infection is a rare occurrence or due to the limitations of the detection methods (Barbut et al., 2000; Behroozian et al., 2013). More recently, Hell et al. (2011); van den Berg et al. (2005) investigated 5 or fewer colonies from each sample and found that $1 / 11(9.1 \%)$ and $2 / 23(8.7 \%)$ of samples, respectively, had multiple strains of $C$. difficile.

With advancing technology, improved sampling methods and sophisticated genotyping, C. difficile transmissions leading to mixed infection and the presence of different toxigenic C. difficile strains are being more readily identified (Eyre et al., 2012, 2013). However, the reported rate of mixed infection remains similar.

\section{CONCLUSION}

Knowledge and understanding of $C$. difficile has grown considerably since George and colleagues made the link

\section{REFERENCES}

Baines, S. D., Crowther, G. S., Todhunter, S. L., Freeman, J., Chilton, C. H., Fawley, W. N., et al. (2013). Mixed infection by Clostridium difficile in an in vitro model of the human gut. J. Antimicrob. Chemother. 68, 1139-1143. doi: $10.1093 /$ jac/dks529

Balmer, O., and Tanner, M. (2011). Prevalence and implications of multiple-strain infections. Lancet Infect. Dis. 11, 868-878. doi: 10.1016/S1473-3099(11)70241-9

Barbut, F., Richard, A., Hamadi, K., Chomette, V., Burghoffer, B., and Petit, J.-C. (2000). Epidemiology of recurrences or reinfections of clostridium difficileassociated diarrhea. J. Clin. Microbiol. 38, 2386-2388.

Behroozian, A. A., Chludzinski, J. P., Lo, E. S., Ewing, S. A., Waslawski, S., Newton, D. W., et al. (2013). Detection of mixed populations of Clostridium difficile from symptomatic patients using capillary-based polymerase chain reaction ribotyping. Infect. Control Hosp. Epidemiol. 34, 961-966. doi: 10.1086/671728

Berry, C. E., Davies, K. A., Owens, D. W., and Wilcox, M. H. (2017). Is there a relationship between the presence of the binary toxin genes in Clostridium difficile strains and the severity of C. difficile infection (CDI)? Eur. J. Clin. Microbiol. Infect. 36, 2405-2415. doi: 10.1007/s10096-017-3075-8

Bidet, P., Barbut, F., Lalande, V., Burghoffer, B., and Petit, J. C. (1999). Development of a new PCR-ribotyping method for Clostridium difficile based on ribosomal RNA gene sequencing. FEMS Microbiol. Lett. 175, 261-266. doi: 10.1111/j.1574-6968.1999.tb13629.x

Borriello, S. P., and Honour, P. (1983). Concomitance of cytotoxigenic and noncytotoxigenic clostridium difficile in stool specimens. J. Clin. Microbiol. 18, 1006-1007. between C. difficile and human diseases (Heinlen and Ballard, 2010). Evidence of dual strain infection was first identified by Borriello and Honour (1983) after observing differential expression of toxins (Borriello and Honour, 1983). With advancing technology, C. difficile typing methods are becoming more discriminative and therefore mixed strain infection can be detected more readily. Interestingly, the incidence of mixed strain C. difficile infection has been stable.

Currently, there are no studies examining the role of mixed or dual strain infection with $C$. difficile in human beings. A previous study in hamsters suggests that mixed strain infection has the potential to be both beneficial and harmful depending on the nature of the infecting strains (Merrigan et al., 2009). In human beings, it is recognized that patients who are colonized by non-toxigenic $C$. difficile strain is at lower risk of developing C. difficile infection while in hospital. This is supported by results from a Phase 2 randomized controlled trial using non-toxigenic $C$. difficile spores to prevent recurrent $C$. difficile infection (Gerding et al., 2015). Further studies to elucidate the interaction between co-infecting or colonizing and infecting $C$. difficile strains may help identify potential exploitable mechanisms to prevent C. difficile infection.

\section{AUTHOR CONTRIBUTIONS}

MW wrote the manuscript scope and outline, supervised the manuscript writing, and carried out multiple rounds of editing. PD carried out the literature search, wrote the manuscript draft, and carried out multiple rounds of re-writing.

Carter, G. P., Chakravorty, A., Pham Nguyen, T. A., Mileto, S., Schreiber, F., Li, L., et al. (2015). Defining the roles of TcdA and TcdB in localized gastrointestinal disease. Systemic organ damage, and the host response during clostridium difficile infections. mBio 6:e00551. doi: 10.1128/mBio.00551-15

DePestel, D. D., and Aronoff, D. M. (2013). Epidemiology of clostridium difficile infection. J. Pharm. Pract. 26, 464-475. doi: 10.1177/0897190013499521

Devlin, H. R., Au, W., Foux, L., and Bradbury, W. C. (1987). Restriction endonuclease analysis of nosocomial isolates of clostridium difficile. J. Clin. Microbiol. 25, 2168-2172.

Di Bella, S., Ascenzi, P., Siarakas, S., Petrosillo, N., and di Masi, A. (2016). Clostridium difficile toxins $\mathrm{A}$ and $\mathrm{B}$ : insights into pathogenic properties and extraintestinal effects. Toxins 8:134. doi: 10.3390/toxins8050134

Eyre, D. W., Cule, M. L., Griffiths, D., Crook, D. W., Peto, T. E., Walker, A. S., et al. (2013). Detection of mixed infection from bacterial whole genome sequence data allows assessment of its role in Clostridium difficile transmission. PLoS Comput. Biol. 9:e1003059. doi: 10.1371/journal.pcbi.1003059

Eyre, D. W., Walker, A. S., Griffiths, D., Wilcox, M. H., Wyllie, D. H., Dingle, K. E., et al. (2012). Clostridium difficile mixed infection and reinfection. J. Clin. Microbiol. 50, 142-144. doi: 10.1128/JCM.05177-11

Figueroa, I., Johnson, S., Sambol, S. P., Goldstein, E. J. C., Citron, D. M., and Gerding, D. N. (2012). Relapse versus reinfection: recurrent Clostridium difficile infection following treatment with fidaxomicin or vancomycin. Clin. Infect. Dis. 55(Suppl. 2), S104-S109. doi: 10.1093/cid/cis357

Gerding, D. N., Johnson, S., Rupnik, M., and Aktories, K. (2014). Clostridium difficile binary toxin CDT: mechanism, epidemiology, and potential clinical importance. Gut Microbes 5, 15-27. doi: 10.4161/gmic.26854 
Gerding, D. N., Meyer, T., Lee, C., Cohen, S. H., Murthy, U. K., Poirier, A., et al. (2015). Administration of spores of nontoxigenic Clostridium difficile strain M3 for prevention of recurrent $\mathrm{C}$. difficile infection: a randomized clinical trial. JAMA 313, 1719-1727. doi: 10.1001/jama.2015.3725

Ghose, C. (2013). Clostridium difficile infection in the twenty-first century. Emerg. Microbes Infect. 2:e62. doi: 10.1038/emi.2013.62

Goldenberg, J. Z., Ma, S. S. Y., Saxton, J. D., Martzen, M. R., Vandvik, P. O., Thorlund, K., et al. (2013). Probiotics for the prevention of Clostridium difficile-associated diarrhea in adults and children. Cochrane Database Syst. Rev. 12:CD006095. doi: 10.1002/14651858.CD006095.pub3

Heinlen, L., and Ballard, J. D. (2010). Clostridium difficile infection. Am. J. Med. Sci. 340, 247-252. doi: 10.1097/MAJ.0b013e3181e939d8

Hell, M., Permoser, M., Chmelizek, G., Kern, J. M., Maass, M., Huhulescu, S., et al. (2011). Clostridium difficile infection: monoclonal or polyclonal genesis? Infection 39, 461-465. doi: 10.1007/s15010-011-0167-8

Kamboj, M., Khosa, P., Kaltsas, A., Babady, N. E., Son, C., and Sepkowitz, K. A. (2011). Relapse versus reinfection: surveillance of clostridium difficile infection. Clin. Infect. Dis. 53, 1003-1006. doi: 10.1093/cid/ci r643

Kelly, C. P., and LaMont, J. T. (1998). Clostridium difficile infection. Ann. Rev. Med. 49, 375-390. doi: 10.1146/annurev.med.49.1.375

Knetsch, C. W., Lawley, T. D., Hensgens, M. P., Corver, J., Wilcox, M. W., and Kuijper, E. J. (2013). Current application and future perspectives of molecular typing methods to study Clostridium difficile infections. Euro Surveill. 18:20381. doi: 10.2807/ese.18.04.20381-en

Kuijper, E. J., van den Berg, R. J., and Brazier, J. S. (2009). "Comparison of Molecular Typing Methods Applied to Clostridium difficile," in Molecular Epidemiology of Microorganisms: Methods and Protocols, ed. D. A. Caugant (Totowa, NJ: Humana Press), 159-171.

Lyerly, D. M., Saum, K. E., MacDonald, D. K., and Wilkins, T. D. (1985). Effects of Clostridium difficile toxins given intragastrically to animals. Infect. Immunity47, 349-352.

Lyras, D., O'Connor, J. R., Howarth, P. M., Sambol, S. P., Carter, G. P., Phumoonna, T., et al. (2009). Toxin B is essential for virulence of Clostridium difficile. Nature 458, 1176-1179. doi: 10.1038/nature07822

Martin, J. S. H., Monaghan, T. M., and Wilcox, M. H. (2016). Clostridium difficile infection: epidemiology, diagnosis and understanding transmission. Nat. Rev. Gastroenterol. Hepatol. 13, 206-216. doi: 10.1038/nrgastro.2016.25

Merrigan, M. M., Sambol, S. P., Johnson, S., and Gerding, D. N. (2009). New approach to the management of Clostridium difficile infection: colonisation with non-toxigenic C. Difficile during daily ampicillin or ceftriaxone administration. Int. J. Antimicrob. Agents 33(Suppl. 1), S46-S50. doi: 10.1016/ S0924-8579(09)70017-2

O’Neill, G. L., Beaman, M. H., and Riley, T. V. (1991). Relapse versus reinfection with Clostridium difficile. Epidemiol. Infect. 107, 627-635. doi: 10.1017/ S0950268800049323

Samra, Z., Talmor, S., and Bahar, J. (2002). High prevalence of toxin A-negative toxin B-positive Clostridium difficile in hospitalized patients with gastrointestinal disease. Diagn. Microbiol. Infect. Dis. 43, 189-192. doi: 10.1016/ S0732-8893(02)00400-5

Savidge, T. C., Pan, W. H., Newman, P., O'Brien, M., Anton, P. M., and Pothoulakis, C. (2003). Clostridium difficile toxin B is an inflammatory enterotoxin in human intestine. Gastroenterology 125, 413-420. doi: 10.1016/ S0016-5085(03)00902-8

Sharp, J., and Poxton, I. R. (1985). An immunochemical method for fingerprinting Clostridium difficile. J. Immunol. Methods 83, 241-248. doi: 10.1016/00221759(85)90246-7
Sim, J. H. C., Truong, C., Minot, S. S., Greenfield, N., Budvytiene, I., Lohith, A., et al. (2017). Determining the cause of recurrent Clostridium difficile infection using whole genome sequencing. Diagn. Microbiol. Infect. Dis. 87, 11-16. doi: 10.1016/j.diagmicrobio.2016.09.023

Sun, J., Mc Millen, T., Babady, N. E., and Kamboj, M. (2016). Role of coinfecting strains in recurrent clostridium difficile infection. Infect. Control Hosp. Epidemiol. 37, 1481-1484. doi: 10.1017/ice.2016.187

Surawicz, C. M., Brandt, L. J., Binion, D. G., Ananthakrishnan, A. N., Curry, S. R., Gilligan, P. H., et al. (2013). Guidelines for diagnosis, treatment, and prevention of Clostridium difficile infections. Am. J. Gastroenterol. 108, 478-98; quiz 99. doi: 10.1038/ajg.2013.4

Tanner, H. E., Hardy, K. J., and Hawkey, P. M. (2010). Coexistence of multiple multilocus variable-number tandem-repeat analysis subtypes of Clostridium difficile PCR ribotype 027 strains within fecal specimens. J. Clin. Microbiol. 48, 985-987. doi: 10.1128/JCM.02012-09

Tenover, F. C., Åkerlund, T., Gerding, D. N., Goering, R. V., Boström, T., Jonsson, A.-M., et al. (2011). Comparison of strain typing results for clostridium difficile isolates from north america. J. Clin. Microbiol. 49, 1831-1837. doi: 10.1128/ JCM.02446- 10

Tonna, I., and Welsby, P. D. (2005). Pathogenesis and treatment of \&lt;em\&gt;Clostridium difficile\&lt;em\&gt; infection. Postgrad. Med. J. 81, 367-369. doi: 10.1136/pgmj.2004.028480

van den Berg, R. J., Ameen, H. A., Furusawa, T., Claas, E. C., van der Vorm, E. R., and Kuijper, E. J. (2005). Coexistence of multiple PCR-ribotype strains of Clostridium difficile in faecal samples limits epidemiological studies. J. Med. Microbiol. 54(Pt 2), 173-179. doi: 10.1099/jmm.0.45825-0

Vedantam, G., Clark, A., Chu, M., McQuade, R., Mallozzi, M., and Viswanathan, V. K. (2012). Clostridium difficile infection: toxins and non-toxin virulence factors, and their contributions to disease establishment and host response. Gut Microbes 3, 121-134. doi: 10.4161/gmic.19399

Wilcox, M. H., Fawley, W. N., Settle, C. D., and Davidson, A. (1998). Recurrence of symptoms in Clostridium difficile infection-relapse or reinfection? J. Hosp. Infect. 38, 93-100. doi: 10.1016/S0195-6701(98)90062-7

Wilcox, M. H., Gerding, D. N., Poxton, I. R., Kelly, C., Nathan, R., Birch, T., et al. (2017). Bezlotoxumab for prevention of recurrent Clostridium difficile infection. N. Engl. J. Med. 376, 305-317. doi: 10.1056/NEJMoa1602615

Conflict of Interest Statement: MW has received: consulting fees from Actelion, Astellas, bioMerieux, Cambimune, Da Volterra, Ferring, MedImmune, Menarini, Merck, Meridian, Pfizer, Qiagen, Sanofi-Pasteur, Seres, Spero, Summit, Synthetic Biologics, and Valneva; lecture fees from Alere, Astellas, Merck, and Pfizer; and grant support from Actelion, Astellas, bioMerieux, Da Volterra, Merck, Motif Biosciences, Nabriva, Paratek, Pfizer, Sanofi-Pasteur, Seres, and Summit.

The remaining author declares that the research was conducted in the absence of any commercial or financial relationships that could be construed as a potential conflict of interest.

The reviewer SJ declared a past collaboration with one of the authors MW to the handling Editor.

Copyright $\odot 2019$ Dayananda and Wilcox. This is an open-access article distributed under the terms of the Creative Commons Attribution License (CC BY). The use, distribution or reproduction in other forums is permitted, provided the original author(s) and the copyright owner(s) are credited and that the original publication in this journal is cited, in accordance with accepted academic practice. No use, distribution or reproduction is permitted which does not comply with these terms. 